Pacific Journal of Mathematics

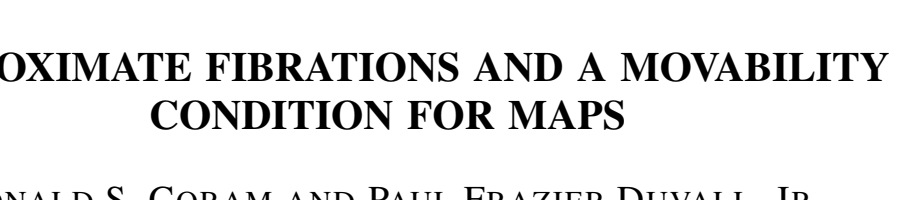




\title{
APPROXIMATE FIBRATIONS AND A MOVABILITY CONDITION FOR MAPS
}

\author{
Donald Coram and Paul Duvall
}

\begin{abstract}
In a previous paper the authors defined the approximate homotopy lifting property and studied its implications. This property is a generalization of the homotopy lifting property of classical fiber space theory. Here a necessary and sufficient condition on point-inverses for a map to have the approximate homotopy lifting property for $n$-cells is given; and the approximate homotopy lifting property for $n$-cells is shown to imply the approximate homotopy lifting property for all spaces. A corollary is that, in a fairly general context, any two point-inverses of a Serre (weak) fibration have the same shape. By combining these results with results of $L$. Husch, some conditions are obtained under which a map between manifolds can be approximated by locally trivial fibrations.
\end{abstract}

1. Introduction and preliminaries. Throughout this paper, $p: E \rightarrow B$ will denote a surjective map between locally compact, separable metric ANR's $E$ and $B$. We say that $p$ has the approximate homotopy lifting property (AHLP) with respect to the space $X$ if whenever $h: X \times I \rightarrow B$ and $\tilde{h}: X \times\{0\} \rightarrow E$ are maps such that $p \tilde{h}=$ $h \mid X \times\{0\}$ and $\varepsilon$ is a cover of $B, h$ extends to a map $\tilde{h}: X \times I \rightarrow E$ such that $h$ and $p \tilde{h}$ are $\varepsilon$-close. By a simple modification of $[4 ; \mathrm{XX}$, 2.4], if $p$ has the AHLP with respect to $X$, we may choose $\widetilde{h}$ to be stationary when $h$ is, i.e., if $h(p(x), t)=p(x)$ for all $t, \tilde{h}(x, t)=x$ for all $t$. If $p$ has the AHLP for all spaces, we say that $p$ is an $a p$ proximate fibration. (It suffices to have the AHLP for metric spaces by [3, Prop. 1.4].)

Approximate fibrations and approximate lifting were introduced in [3] as an abstraction of the useful lifting properties possessed by $U V^{k}$-maps [9], [11], [12]. It is shown in [3] that approximate fibrations have shape theoretic properties analogous to the homotopy theoretic properties of Hurewicz fibrations. For example, under appropriate hypotheses on $E$ and $B$ any two point inverses of $p$ have the same shape, and $p$ induces an exact sequence involving the homotopy groups of $E$ and $B$ and the shape-theoretic homotopy groups of any point inverse of $p$.

In this paper, we study conditions which imply that a map is an approximate fibration. Section 2 is devoted to showing that, in the case of approximate liftings, the difference between Serre and Hurewicz fibrations disappears; that is, the AHLP for all cells is 
equivalent to the AHLP for all spaces. In $\S 3$ we define a movability condition for maps and study its relationship to approximate liftings. Finally, in $\S 4$ we give some applications.

We will use the following notation and terminology. Map means continuous function. All covers are open covers. If $\delta$ is a cover of a space $X$, and $V \in \delta$, the star of $V$ is the union of all members of $\delta$ which intersect $V$. The star of $\delta$ is the cover whose elements are the stars of members of $\delta$. $\delta$ is star-finite if each member of $\delta$ meets only finitely many members of $\delta$. We say that $\delta$ refines the cover $\varepsilon$ and write $\delta<\varepsilon$ if each member of $\delta$ is contained in a member of $\varepsilon$. Also $\delta$ star-refines $\varepsilon$ if the star of $\delta$ refines $\varepsilon$. We will often use the fact that each open cover of a separable metric space has a star-finite star refinement [4, p. 167 and p. 255]. If $\varepsilon$ is a cover of the space $X$ and $f, g: Y \rightarrow X$ are maps, we say $f$ and $g$ are $\varepsilon$-close provided that for each $y \in Y$ there is $V \in \varepsilon$ such that $f(y), g(y) \in V$. Also $f$ and $g$ are $\varepsilon$-homotopic if there is a homotopy $H$ between $f$ and $g$ such that for each $y \in Y$, there is a $V \in \varepsilon$ such that $H(\{y\} \times I) \subset V$. If $\varepsilon$ is a cover of $B$ and $f: X \rightarrow E, g: X \rightarrow B$ are maps such that $p f$ and $g$ are $\varepsilon$-close, we say that $f$ is an $\varepsilon$-lift of $g$. If $\varepsilon$ is a positive number, $\varepsilon$-close means close with respect to the cover by open $\varepsilon$-balls. Similar definitions hold for $\varepsilon$-homotopic, $\varepsilon$-lift. If $C$ is a subset of a space $X$, a neighborhood of $C$ is a set which contains $C$ in its interior. If $x$ is a point in a metric space $X$ with metric $d, N(x, r)=\{y \in X \mid d(x, y)<r\}$. If $x$ is a vertex of a complex $K, \operatorname{st}(x, K)=U\{\operatorname{int} \sigma \mid x$ is a vertex of $\sigma\}$. For $b \in B, F_{b}$ denotes $p^{-1}(b)$. In discussing homotopies defined on $X \times I$ we often identify $X \times\{0\}$ with $X$.

We conclude this section with several lemmas which are analogous to standard facts in the usual theory of fibrations. Since they will be used in a crucial way in the rest of the paper, we include indications of proofs for completeness.

Lemma 1.1 (see [3], Lemma 1.2). Suppose that $p$ has the AHLP for the metric space $X$. Given a cover $\varepsilon$ of $B$ there is a refinement $\delta$ of $\varepsilon$ such that if $h: X \times I \rightarrow B$ and $\tilde{h}: X \rightarrow E$ are maps such that $\tilde{h}$ is $\delta$-lift of $h \mid X \times\{0\}, \tilde{h}$ extends to an $\varepsilon$-lift of $h$.

Proof. Let $\omega$ be a twice star refinement of $\varepsilon$ and let $\delta$ be a star refinement of $\omega$ such that any two $\delta$-close maps into $B$ are $\omega$ homotopic. Let $q: X \rightarrow(0,1)$ be a map such that for each $x \in X$, $h(x \times[0, q(x)])$ is contained in some member of $\delta$. Let $\phi: X \times$ $[-1,0] \rightarrow B$ be an $\omega$-homotopy between $p \tilde{h}$ and $h \mid X \times 0$. Let $g: X \times[-1,1] \rightarrow B$ be given by $g(x, t)=\left\{\begin{array}{l}\phi(x, t),-1 \leqq x \leqq 0 \\ h(x, t), 0 \leqq x \leqq 1 .\end{array}\right.$ There 
is a $\widetilde{g}: X \times[-1,1] \rightarrow E$ which extends $\tilde{h}$ and is an $\omega$-lift of $g$ by the AHLP. Define $\tilde{h}: X \times I \rightarrow E$ by

$$
\widetilde{h}(x, t)= \begin{cases}\widetilde{g}\left(x, \frac{2 t}{q(x)}-1\right), & \text { if } \quad 0 \leqq t \leqq \frac{q(x)}{2} \\ \widetilde{g}(x, 2 t-q(x)), & \text { if } \frac{q(x)}{2} \leqq t \leqq q(x) \\ \widetilde{g}(x, t), & \text { if } q(x) \leqq t \leqq 1 .\end{cases}
$$

We need to show that $\tilde{h}$ is an $\varepsilon$-lift of $h$. If $(x, t)$ is a point in $X \times I$ with $t \leqq q(x) / 2$, then $p \widetilde{h}(x, t)=p \widetilde{g}(x, 2 t / q(x)-1)$ which is $\omega$-close to $g(x, 2 t / q(x)-1)=\phi(x, 2 t / q(x)-1) . \quad \phi(x, 2 t / q(x)-1)$ is $\omega$-close to $\phi(x, 0)=h(x, 0)$, and $h(x, 0)$ is $\omega$-close to $h(x, t)$ by our choice of $q(x)$. Since $\omega$ twice star refines $\varepsilon, p \tilde{h}(x, t)$ is $\varepsilon$-close to $h(x, t)$.

If $q(x) / 2 \leqq t \leqq q(x)$, then $y=2 t-q(x) \in[0, q(x)]$, so $p \tilde{h}(x, t)=$ $p \widetilde{g}(x, y)$ which is $\omega$-close to $g(x, y)=h(x, y)$, which is $\omega$-close to $h(x, t)$ by our choice of $q(x)$, so $p \tilde{h}(x, t)$ is $\varepsilon$-close to $h(x, t)$. If $t>q(x), p \tilde{h}(x, t)$ is $\omega$-close to $g(x, t)=h(x, t)$.

Lemma 1.2. Suppose that $p$ has the AHLP for $I^{q}, q \leqq k$ and let $(X, A)$ be a polyhedral pair with dimension $X \leqq k$. Then given a cover $\varepsilon$ of $B$ there is a cover $\delta$ of $B$ such that if $h: X \times I \rightarrow B$ and $\tilde{h}: X \times\{0\} \cup A \times I \rightarrow E$ are maps such that $p \tilde{h} \mid X \times\{0\}=$ $h \mid X \times\{0\}$ and $\widetilde{h} \mid A \times I$ is $\delta$-lift of $h \mid A \times I, \tilde{h}$ extends to an $\varepsilon$-lift of $h$.

Proof. Given $\varepsilon$, let $\delta=\delta_{k}<\delta_{k-1}<\cdots<\delta_{0}=\varepsilon$ be a collection of covers so that $\delta_{i}$ plays the role of $\delta$ in 1.1 for $\varepsilon=\delta_{i-1}, i>0$. Triangulate $X$ so that $A$ is a subcomplex and build the extension over the cells of $X-A$ as in [19,7.2.6], using Lemma 1.1 to extend.

CoRollary 1.3. If $p$ has the AHLP for $I^{q}, q \leqq k$, then $p$ has the AHLP for all polyhedra of dimension $k$ or less.

Note that the $\delta$ in Lemma 1.1 depends only on the cover $\varepsilon$, and on the dimension of $X$ and $\varepsilon$ in Lemma 1.2.

Now let $B^{I}$ denote the space of paths in $B$ with the compact open topology, and let $\Delta_{p}=\left\{(\alpha, e) \in B^{I} \times E \mid p(e)=\alpha(0)\right\}$. Just as in the case of the usual theory of fibrations [4, XX], we have the following characterization for approximate fibrations.

LEMMA 1.4. $p$ is an approximate fibration if and only if $p$ has the AHLP for $\Delta_{p}$.

Sketch of proof. Necessity is clear. For the converse, observe 
that approximate fibrations can be described in terms of approximate path lifting functions [3], whose existence is equivalent to the AHLP for $\Delta_{p}$.

II. The equivalence of weak and strong approximate fibrations. In this section, we will show that the AHLP for finite polyhedra implies the AHLP for all spaces. For the time being, let us say that $p$ is a weak approximate fibration if $p$ satisfies the AHLP for $I^{q}$ for all $q<\infty$.

THEOREM 2.1. Suppose that $p$ is a weak approximate fibration. Then $p$ has the AHLP for all countable locally finite polyhedra.

Proof. Let $X$ be a countable (noncompact) locally finite polyhedron, and let $h: X \times I \rightarrow B, \widetilde{h}: X \rightarrow E$ be maps such that $p \tilde{h}=h \mid X$. Write $X=\left(\bigcup_{i=0}^{\infty} A_{i}\right) \cup\left(\bigcup_{i=0}^{\infty} B_{i}\right)$ such that

(1) $B_{0}=\varnothing, B_{i} \neq \varnothing, A_{j} \neq \varnothing$ for $i \geqq 1, j \geqq 0$,

(2) each $A_{i}, B_{i}$ is a compact polyhedron,

(3) $\left(B_{i} \cap \bigcup_{j=0}^{\infty} A_{j}\right) \subset A_{i-1} \cup A_{i}$ for $i \geqq 1$,

(4) $A_{i} \cap A_{j}=\varnothing$ and $B_{i} \cap B_{j}=\varnothing$ if $i \neq j$.

Given a cover $\varepsilon$ of $B$, let $\delta_{i}$ be the cover of $B$ promised in Lemma 1.2 for $\varepsilon$ and the pair $\left(B_{i}, B_{i} \cap\left(A_{i-1} \cup A_{i}\right)\right)$ for each $i$, and let $\eta_{i}$ be a common refinement of $\delta_{i}$ and $\delta_{i+i}$. There is an extension of $\widetilde{h}$ to $\left(\bigcup_{i=0}^{\infty} A_{i}\right) \times I$ such that $\tilde{h} \mid A_{i} \times I$ is an $\eta_{i}$-lift of $h \mid A_{i} \times I$. By Lemma 1.2 , we can now extend $\widetilde{h}$ over each of the $B_{i}$ 's to an $\varepsilon$-lift of $h$ defined on all of $X \times I$.

THEOREM 2.2. Suppose that $p$ has the AHLP for $I^{q}$ for all $q \leqq k \leqq \infty$. Then $p$ has the AHLP for all separable metric spaces of dimension $k$ or less.

Before proving 2.2, we need to develop some terminology and several lemmas. The strategy of proof is clear. Since we are only concerned with approximate liftings, we can use nerves of covers and canonical maps to translate a lifting problem for metric spaces into one involving polyhedra. For a discussion of nerves and canonical maps, see [1] or [7]. Our first lemma is a restatement of [7, Theorem 8.1].

LEMMA 2.3. Let $Y$ be an ANR, $\omega$ a cover of $Y$, and $f: X \rightarrow Y$ a map, where $X$ is metric. Then $X$ has a cover $\pi$ such that if $\xi$ is any locally finite refinement of $\pi$ and $N_{\xi}$ is the nerve of $\xi$, there is a map $\psi_{\xi}:\left|N_{\xi}\right| \rightarrow Y$ such that $f$ and $\psi_{\xi} \dot{\phi}_{\xi}$ are $\omega$-homotopic in $Y$ for any canonical map $\phi_{\xi}: X \rightarrow\left|N_{\xi}\right|$. 
The map $\psi_{\xi}$ is called a bridge map for $f$ relative to $\omega$.

LEMMA 2.4. Let $X$ be a metric space and let $\omega$ be a cover of $X \times I$. Then there is a star-finite refinement $\xi$ of $\omega$ whose nerve is a triangulation of $P \times I$, where $P$ is the carrier of the nerve of $\xi \mid X$. Furthermore, there is a canonical map $\phi: X \times I \rightarrow P \times I$ for $\xi$ such that $\phi \mid X: X \rightarrow P$ is a canonical map for $\xi \mid X$.

Proof. By [6, IX, 5.6], we may assume that $\omega$ is a cover whose elements are of the form $V \times J$ where the $V$ are the elements of a star-finite cover $\gamma$ of $X$ and $J$ is a subinterval of $I$. Let $K$ be the nerve of $\gamma$, let $P=|K|$, let $g: X \rightarrow P$ be the barycentric map with respect to $\gamma[1, \mathrm{p} .76]$, and let $\phi: X \times I \rightarrow P \times I$ be defined by $\phi(x, t)=(g(x), t)$. Define the cover $\rho$ of $P \times I$ by $\dot{s} t(q, K) \times J \in \rho$ if and only if $V \times J \in \omega$ and $q$ is the vertex of $K$ corresponding to $V$. Choose a subdivision $L$ of $P \times I$ such that $\left\{\operatorname{sit}^{\circ}(q, L) \mid q\right.$ a vertex of $L\}$ refines $\rho$. Then it is easy to check that $\phi$ and $\xi=\left\{\phi^{-1}(s t(q, L)) \mid q\right.$ is a vertex of $L\}$ satisfy the conclusions of lemma.

The next lemma is proved by a slight alteration of the proof of 1.1. We omit the proof.

Lemma 2.5. Suppose $X$ is a metric space and that $p$ satisfies that following condition: given covers $\delta$ and $\varepsilon$ of $E$ and $B$ and maps $h: X \times I \rightarrow B$ and $g: X \rightarrow E$ with $p g=h \mid X$, there is an $\varepsilon$-lift $\tilde{h}$ of $h$ such that $\tilde{h} \mid X$ is $\delta$-close to $g$. Then $p$ has the AHLP with respect to $X$.

Proof of 2.2. We will apply Lemma 2.5. Suppose that $\varepsilon, \delta, g$, and $h$ are given as above. Let $\eta^{\prime}$ be a star refinement of $\varepsilon$. Let $\eta$ be a star refinement of $\eta^{\prime}$ which is fine enough to play the role of $\delta$ in Lemma 1.1 for $\eta^{\prime}$. Let $\omega_{B}$ be a star refinement of $\eta$. Refine the cover $p^{-1} \omega_{B}$ by a cover $\omega_{E}$ which is also a star refinement of $\delta$. Let $\pi_{E}\left(\pi_{B}\right)$ be a cover of $X(X \times I)$ obtained from applying Lemma 2.3 to $\omega_{E}$ and $g\left(\omega_{B}\right.$ and $\left.h\right)$, let $\pi_{E} \times I$ be the cover of $X \times I$ defined by $\pi_{E} \times I=\left\{V \times I \mid V \in \pi_{E}\right\}$, and let $\xi, \phi, P$ be as promised be Lemma 2.4 for some common refinement of $\pi_{E} \times I$ and $\pi_{B}$. We have the diagram

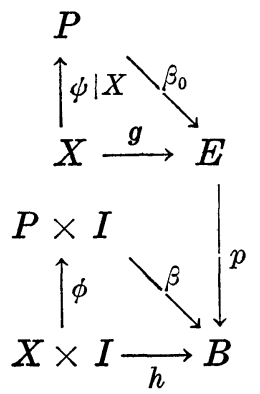


where $\beta_{0}, \beta$ are bridge maps. By the construction, $p \beta_{0}$ and $\beta \mid P$ are $\eta$-close, so $\beta$ has an $\eta^{\prime}$-lift $\widetilde{\beta}$ extending $\beta_{0}$ by Lemma 1.1. Then $\widetilde{\beta} \phi$ is an $\varepsilon$-lift of $h$ such that $g$ and $\widetilde{\beta} \phi \mid X$ are $\delta$-close, so the proof is complete.

The situation is now as follows. The proof of Theorem 2.2 says that when we have the AHLP for the nerves of a cofinal family of covers of a metric space $X$, we have the AHLP for $X$ itself. Theorem 2.1 says that the AHLP for cells implies the AHLP for countable polyhedra. We can put these together to get

THEOREM 2.6. $\quad p$ is a weak approximate fibration if and only if it is an approximate fibration.

Proof. Suppose $p$ is weak approximate fibration. Since $E, B$ are second countable, $B^{I} \times E$ is second countable [4], so $A_{p}$ is a second countable metric space. By 2.1 and $2.2 p$ has the AHLP for $\Delta_{p}$, so the theorem follows by 1.4 .

We now obtain a corollary about Serre fibrations (weak fibrations in [19]). It is known that any two fibers of a Serre fibration over a path connected base have the same weak homotopy type [19, Cor. 7.8.5]. However, the $\sin (1 / x)$-circle has the same weak homotopy type as a point. Could these sets be fibers of a Serre fibration? The corollary answers this question negatively in our setting by showing that a stronger relationship holds between the fibers.

COROLlaRY 2.7. If $p$ is a Serre fibration, $p$ is an approximate fibration. If, in addition, $B$ is path connected and $p$ is a proper map, any two fibers of $p$ have the same shape.

Proof. The first conclusion is immediate from 2.6. The second follows from [3].

III. Movability for maps and approximate lifting. If $p$ is a cell-like map, it follows from [12] that $p$ is an approximate fibration with no further assumptions. As soon as the fibers of $p$ have nontrivial shapes, however, it is clear that some consistency conditions must be placed on the fibers if $p$ is to be an approximate fibration. In this section, we characterize approximate fibrations in terms of a movability condition which is reminiscent of the notions of regularity that have been used to advantage in the study of fibrations [5], [13], [17], [18].

Let $F$ be a compactum in the space $E, x \in F$, and $\omega$ the inverse 
system of neighborhoods of $F$ in $E$ with inclusions as bonding maps. Define $\pi_{k}(F, x)=\stackrel{\lim }{\longleftarrow}\left\{\pi_{k}(U, x) \mid U \in \omega\right\}$ for each $k \geqq 0$. If $V \subset U$ are neighborhoods of $F$ and the projection $\pi_{k}(F, x) \rightarrow \pi_{k}(U, x)$ is an isomorphism onto the image of the inclusion induced map $\pi_{k}(V, x) \rightarrow$ $\pi_{k}(U, x)$ for every $x \in F$, we say that $\pi_{k} F$ is realized as the image of $\pi_{k} V$ in $\pi_{k} U$. This would occur for example if $\pi_{k}(V, x)$ and $\pi_{k}(U, x)$ were the first two terms in a constant inverse sequence with inverse limit $\pi_{k}(F, x)$ for each $x \in F$. A proper map $p: E \rightarrow B$ is a $k$-movable map provided that for each $b \in B$ and each neighborhood $U_{0}$ of the fiber $F_{b}$ there are neighborhoods $U$ and $V$ of $F_{b}$ such that $V \subset U \subset U_{0}$ and $\pi_{i} F_{c}$ is realized as the image of $\pi_{i} V$ in $\pi_{i} U, 0 \leqq i \leqq k$, for every fiber $F_{c} \subset V$. The next lemma gives a useful consequence of $k$-movability.

Lemma 3.1. Suppose $p: E \rightarrow B$ is a k-movable map, $k \geqq 1$. Let $b \in B, U_{0}$ be an arbitrary neighborhood of $F_{b}$, and $U$ and $V$ be neighborhoods of $F_{b}$ in $U_{0}$ such that, for any $F_{c} \subset V, \underline{\pi}_{i} F_{c}$ is realized as the image of $\pi_{i} V$ in $\pi_{i} U, 0 \leqq i \leqq k$. Then for any fiber $F_{c} \subset V$ and any neighborhood $W_{0}$ of $F_{0}$ in $V$, there exist neighborhoods $W$ and $Z$ of $F_{c}$ in $W_{0}$ such that $\alpha_{\sharp}: \pi_{i}(V, Z) \rightarrow \pi_{i}(U, W), 1 \leqq i \leqq k$, is the zero homomorphism where $\alpha:(V, Z) \rightarrow(U, W)$ is inclusion.

Proof. Since $p$ is a $k$-movable map, there are neighborhoods $W$ and $Z$ of $F_{c}$ in $W_{0}$ such that $\pi_{i} F_{c}$ is realized as the image of $\pi_{i} Z$ in $\pi_{i} W, 0 \leqq i \leqq k$. Let $\phi: Z \rightarrow W, \chi: W \rightarrow V$, and $\psi: V \rightarrow U$ be the inclusion maps. By the choice of $Z$ and $V,(\psi \chi)_{\sharp}: \operatorname{im} \phi_{\sharp} \rightarrow \operatorname{im} \psi_{\#}$ is an isomorphism on $\pi_{i}$ for $0 \leqq i \leqq k$. In particular $\chi_{\ddagger}: \pi_{i-1} W \rightarrow \pi_{i-1} V$ is monic on $\operatorname{im} \phi_{\sharp}$, and image $(\psi \chi)_{\sharp}=$ image $\psi_{\sharp}$. Consider the following diagram in which the vertical arrows are inclusion induced and the horizontal arrows are part of the exact homotopy sequence of a pair.

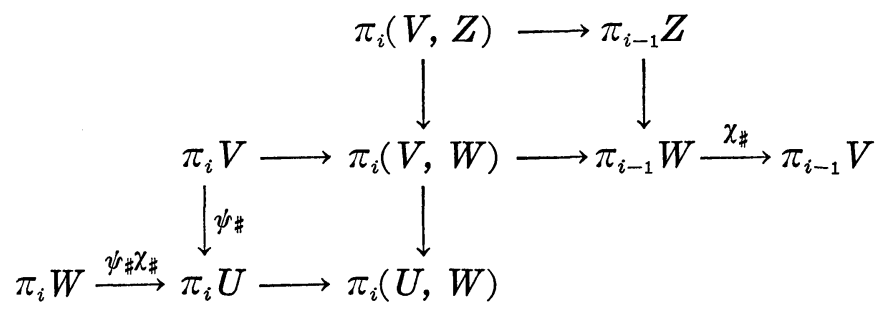

It is an easy "diagram chasing" argument to prove that $\alpha_{\#}$ is the zero homomorphism.

Following Kozlowski and Segal [10] we define a metric compactum $F$ to be $k$-movable $(k \geqq 0)$ if for any $\operatorname{ANR}$ sequence $\left\{X_{i}, p_{i j}\right\}$ 
associated with $F$ given $i$ there exists $j \geqq i$ such that for any mapping $f_{0}: K \rightarrow X_{j}$ from a $k$-complex and for any $l \geqq j$, there is a map $f_{1}: K \rightarrow X_{l}$ such that $p_{i j} f_{0} \simeq p_{i l} f_{1}$.

LEMMA 3.2. Let $p: E \rightarrow B$ be a proper map. If $p$ is a k-movable map, then each fiber $F$ is a k-movable compactum.

Proof. We choose the ANR sequence $\left\{X_{i}\right\}$ associated with $F$ to be a nested sequence of compact ANR neighborhoods of $F \times\{q\}$ in $E \times Q$ with the inclusions as bonding maps. (See [3], $Q$ denotes the Hilbert cube.) The proof is by induction on $k$. Let $k=0$. Given $i$, choose open sets $U$ and $V$ in $E$ and a connected open set $Q_{i} \subset Q$ such that

$$
F \times\{q\} \subset V \times Q_{i} \subset U \times Q_{i} \subset X_{i}
$$

and $\pi_{0} F$ is realized as the image of $\pi_{0} V$ in $\pi_{0} U$. Choose $j$ so that $X_{j} \subset V \times Q_{i}$. Suppose $f: K \rightarrow X_{j}$ is a map from a 0 -complex $K$ and $l \geqq j$. Select open sets $W$ and $Z$ in $E$ and $Q_{l}$ in $Q$ such that

$$
F \times\{q\} \subset Z \times Q_{l} \subset W \times Q_{l} \subset X_{l}
$$

and $\pi_{0} F$ is realized as the image of $\pi_{0} Z$ in $\pi_{0} W$. For each $x$ in $K$ the first coordinate of $f(x)$ can be connected by a path in $U$ to a point in $Z$ by the $\pi_{0}$ realization statements, and the second coordinate of $f(x)$ can be connected to $q$ by the path connectivity of $Q_{i}$. Since $U \times Q_{i} \subset X_{i}, f$ extends to $\hat{f}: K \times I \rightarrow X_{i}$ such that $\hat{f}(x, 1) \in Z \times$ $Q_{l} \subset X_{l}$.

Now suppose $k>0$. Given $i$, choose open sets $U$ and $V$ in $E$ and a contractible open set $Q_{i}$ in $Q$ such that

$$
F \times\{q\} \subset V \times Q_{i} \subset U \times Q_{i} \subset X_{i}
$$

and $\pi_{k} F$ is realized as the image of $\pi_{k} V$ in $\pi_{k} U$. Choose $i^{\prime}$ such that $X_{i^{\prime}} \subset V \times Q_{i^{\prime}}$, and choose $j \geqq i^{\prime}$ by induction so that for $l^{\prime} \geqq j$ each map of a $(k-1)$-complex into $X_{j}$ is homotopic in $X_{i^{\prime}}$ to a map of the complex into $X_{l^{\prime}}$. Suppose $f: K \rightarrow X_{j}$ is a map from a $k$ complex $K$ and $l \geqq j$. Select open sets $Z$ and $W$ in $E$ and a contractible open set $Q_{l}$ in $Q$ such that

$$
F \times\{q\} \subset Z \times Q_{l} \subset W \times Q_{l} \subset X_{l}
$$

and the inclusion induced homomorphism $\alpha_{\ddagger}: \pi_{k}(V, Z) \rightarrow \pi_{k}(U, W)$ is zero. Choose $l^{\prime}$ such that $X_{l^{\prime}} \subset Z \times Q_{l}$. Then $f$ extends to $f: K \times\{0\}$ $\cup K^{k-1} \times I \rightarrow X_{i^{\prime}}$ such that $f(x, 1) \in X_{l^{\prime}}$ for each $x \in K^{k-1}$. For each simplex $A$ in $K, f \mid(A \times\{0\} \cup \operatorname{Bd} A \times I)$ defines an element of 
$\pi_{k}\left(V \times Q_{i}, Z \times Q_{l}\right)$ which maps to zero in $\pi_{k}\left(U \times Q_{i}, W \times Q_{l}\right)$ since $\alpha_{\#}$ is zero and $Q_{i}$ and $Q_{l}$ are contractible. Hence $f$ extends to $\hat{f}: K \times I \rightarrow U \times Q_{i} \subset X_{i}$ and $f(x, 1) \in W \times Q_{l} \subset X_{l}$ for each $x \in K$ as desired to complete the proof.

On the other hand, the converse of Lemma 3.2 is false. For example define $f: S^{k} \times B^{k+1} \rightarrow B^{k+1}$ by $f(x, y)=|y| x$ where $x \in S^{k}$, $y \in B^{k+1}$ and $|y|$ denotes the norm of $y$. Each point-inverse of $f$ is homeomorphic to $S^{k}$ which is $k$-movable compactum, but $f$ fails to be a $k$-movable map at $f^{-1}(0)$.

THEOREM 3.3. If $p: E \rightarrow B$ is a k-movable map, then $p$ has the approximate homotopy lifting property for $I^{i}, 0 \leqq i \leqq k$.

Proof. The proof is by induction on $k$. We thus first take $k=0$. Let $g:\{0\} \rightarrow E$ and $G:\{0\} \times I \rightarrow B$ be given maps such that $p g(0)=G(0,0)$, and let $\varepsilon>0$ be a given number. For each $b \in B$, choose $U_{b} \subset p^{-1}(N(b, \varepsilon / 2))$ and an open set $V_{b}$ such that $F_{b} \subset V_{b} \subset U_{b}$ and $\pi_{0} F_{c}$ is realized as the image of $\pi_{0} V_{b}$ in $\pi_{0} U_{b}$ for every fiber $F_{c} \subset V_{b}$. Choose a finite subcollection $\omega$ of $\left\{V_{b} \mid b \in B\right\}$ such that $\omega$ covers $p^{-1} G(I)$. Let $0=t_{0}<t_{1}<\cdots<t_{n}=1$ be a partition of $I$ such that the image of each subinterval $p^{-1} G\left[t_{i}, t_{i+1}\right]$ lies in some element of $\omega$ which we denote $V_{i}$. (These need not be distinct.) Suppose $g$ has been extended to $G_{j}:\left[0, t_{j}\right] \rightarrow E, 0 \leqq j \leqq n-1$ such that $G_{j}\left(\left[t_{i}, t_{i+1}\right]\right) \subset U_{i}$ for $0 \leqq i \leqq j-1$, and $G_{j}\left(t_{j}\right) \in V_{j}$. $\quad\left(G_{0}=g\right.$ begins this induction.) If $j<n-1$, let $W_{j}=V_{j} \cap V_{j+1}$; and if $j=n-1$ let $W_{j}=V_{j}$. Choose an open set $Z_{j}$ such that $p^{-1}\left(G\left(t_{j+1}\right)\right) \subset Z_{j} \subset W_{j}$ and $\pi_{0} p^{-1}\left(G\left(t_{j+1}\right)\right)$ is realized as the image of $\pi_{0}\left(Z_{j}\right)$ in $\pi_{0}\left(W_{j}\right)$. Hence, every point in $V_{j}$ can be joined by a path in $U_{j}$ to a point in $Z_{j}$. Thus, $G_{j}$ can be extended to $G_{j+1}:\left[0, t_{j+1}\right] \rightarrow E$ such that

$$
G_{j+1}\left(\left[t_{j}, t_{j+1}\right]\right) \subset U_{j}
$$

and

$$
G_{j+1}\left(t_{j+1}\right) \in V_{j+1} \text {. }
$$

Let $\widetilde{G}=G_{n}:[0,1] \rightarrow E$. Then $\widetilde{G}$ extends $g$; and if $t \in\left[t_{i}, t_{i+1}\right]$, then $G(t) \in p\left(V_{i}\right) \subset p\left(U_{i}\right)$ and $p \widetilde{G}(t) \in p\left(U_{i}\right)$, so $d(p \widetilde{G}, G)<\varepsilon$ by the choice of $U_{i}$.

Now assume $k>0$ and that the theorem is true for integers less than $k$. Let $g: I^{k} \rightarrow E$ and $G: I^{k} \times I \rightarrow B$ be given maps such that $p g(t)=G(t, 0)$, and let $\varepsilon>0$ be a given number. For each $b \in B$ choose $U_{b} \subset p^{-1}(N(b, \varepsilon / 2))$ and an open set $V_{b}$ such that $F_{b} \subset$ $V_{b} \subset U_{b}$ and $\pi_{k} F_{c}$ is realized as the image of $\pi_{k} V_{b}$ in $\pi_{k} U_{b}$ for every fiber $F_{c} \subset V_{b}$. Choose a finite subcollection $\omega$ of $\left\{V_{b} \mid b \in B\right\}$ which 
covers $p^{-1} G\left(I^{k} \times I\right)$. Subdivide $I^{k} \times I$ into rectangles each one of whose images is contained in some $V_{b} \in \omega$. We order the collection of rectangles lexicographically and denote the result as $\left\{R_{i} \mid i=1,2\right.$, $\cdots, n\}$. For each rectangle $R_{i}$ choose an element of $\omega$ denoted $V_{i}$ such that $p^{-1} G\left(R_{i}\right) \subset V_{i}$. (These need not be distinct.) Each $R_{i}=$ $J_{i} \times\left[s_{i}, t_{i}\right]$ where $J_{i}$ is a rectangle in $I^{k}$ and $\left[s_{i}, t_{i}\right] \subset I$. Let $m_{i}=$ $\left(m_{i}^{\prime}, t_{i}\right)$ where $m_{i}^{\prime}$ is the center of $J_{i}$. If $t_{i} \neq 1, m_{i} \in R_{i} \cap R_{j}$ for some $j>i$. In this case define $W_{i}=V_{i} \cap V_{j}$. If $t_{i}=1$, let $W_{i}=V_{i}$. Now choose a neighborhood $Z_{i}$ of $p^{-1} G\left(m_{i}\right)$ in $W_{i}$ such that the conclusion of Lemma 3.1 holds. There is a number $\zeta>0$ such that if $e \in E$ and $d\left[p(e), G\left(J_{i} \times\left\{t_{i}\right\}\right)\right]<\zeta$ for some $i$ then $e \in W_{i}$. Also, there is another number $\eta>0$ such that if $e \in E$ and $d\left[p(e), G\left(m_{i}\right)\right]<\eta$ for some $i$ then $e \in Z_{i}$. Corresponding to $\varepsilon_{1}=\min \{\eta / 2, \zeta\}$, there is a $\delta_{1}$ satisfying the conclusion to Lemma 1.1.

Let $A=I^{k} \times\{0\} \cup\left\{\mathrm{Bd} J_{i} \times I \mid i=1,2, \cdots, n\right\}$. By the inductive hypothesis $g$ can be extended to $G^{\prime}: A \rightarrow E$ such that $G^{\prime}\left(R_{i} \cap A\right) \subset V_{i}$ for each $i$ and $d\left(p G^{\prime}, G \mid A\right)<\min \left\{\delta_{1}, \zeta\right\}$. Now suppose $G^{\prime}$ has been extended to $G_{j}^{\prime}: A \cup\left\{R_{i} i=1,2, \cdots, j-1\right\}$ such that $G_{j}^{\prime}\left(R_{i}\right) \subset U_{i}$ and $G_{j}^{\prime}\left(J_{i} \times\left\{t_{i}\right\}\right) \subset W_{i}$ for $1 \leqq i \leqq j-1$. Let $S_{j}$ be a rectangle in $J_{j} \times\left\{t_{j}\right\}$ containing $m_{j}$ such that diam $G\left(S_{j}\right)<\eta / 2$. By the inductive hypothesis and the choice of $\delta_{1}, G_{j}^{\prime}$ extends to $G_{j}^{\prime \prime}: A \cup\left\{R_{i}=1,2, \cdots\right.$, $j-1\} \cup\left(\operatorname{Bd} R_{j}-\operatorname{Int} S_{j}\right) \rightarrow E$ such that $d\left[p G_{j}^{\prime \prime}(x), G(x)\right]<\varepsilon_{1}$ for $x \in J_{j} \times\left\{t_{j}\right\}-\operatorname{Int} S_{j}$. Now $G_{j}^{\prime \prime} \mid \operatorname{Bd} R_{j}-\operatorname{Int} S_{j}$ defines an element of $\pi_{k}\left(V_{j}, Z_{j}\right)$ since if $x \in \mathrm{Bd} S_{j}$ then

$$
d\left[p G_{j}^{\prime \prime}(x), G\left(m_{j}\right)\right] \leqq d\left[p G_{j}^{\prime \prime}(x), G(x)\right]+d\left[G(x), G\left(m_{j}\right)\right]<\eta .
$$

Hence by Lemma 3.2, $G_{j}^{\prime \prime}$ extends to $G_{j+1}^{\prime}: A \cup\left\{R_{i} i=1,2, \cdots, j\right\} \rightarrow E$ such that $G_{j+1}^{\prime}\left(R_{j}\right) \subset U_{j}$ and $G_{j+1}^{\prime}\left(J_{j} \times\left\{t_{j}\right\}\right) \subset W_{j}$.

The map $\widetilde{G}=G_{n+1}^{\prime}: I^{k} \times I \rightarrow E$ is the desired map since $\widetilde{G}$ extends $g$ and if $t \in R_{i}$ then both $G(t)$ and $p \widetilde{G}(t)$ are elements of $p\left(U_{i}\right)$ so $d(p \widetilde{G}, G)<\varepsilon$.

CoROllary 3.4. If $p: E \rightarrow B$ is a k-movable map for all $k$, then $p$ is an approximate fibration.

Proof. By Theorem 3.3, $p$ has the approximate homotopy lifting property for cells of all dimensions. Hence by Theorem 2.6, $p$ is an approximate fibration.

Using the argument of [3, Theorem 2.4] we also have the following proposition.

Proposition 3.5. If $p: E \rightarrow B$ is a proper map with the approximate homotopy lifting property for cells of dimension $\leqq k+1$, 
then $p$ is a k-movable map.

REMARK. If the definition of $k$-movability for a map were changed to require only an epimorphism in dimension $k$ rather than an isomorphism, then Theorem 3.3 would still be true as stated and Proposition 3.5 would be true without assuming the approximate homotopy lifting property for cells of dimension $k+1$. This added generality is not however worth the added complication.

We now give another condition on a map, similar to $k$-movability for all $k$ but more geometric, which also implies that the map is an approximate fibration. A proper map $p: E \rightarrow B$ is a completely movable map provided that for each $b \in B$ and each neighborhood $U$ of the fiber $F_{b}$ there is a neighborhood $V$ of $F_{b}$ in $U$ such that if $F_{c}$ is any fiber in $V$ and $W$ is any neighborhood of $F_{c}$ in $V$ then there is a homotopy $H: V \times I \rightarrow U$ such that $H(x, 0)=x$ and $H(x, 1) \in W$ for each $x \in V$ and $H(x, t)=x$ for each $x \in F_{c}$. We say that $V$ is a movability choice for $U$ and $b$.

Proposition 3.6. Let $p: E \rightarrow B$ be a proper map. Then $p$ is completely movable if and only if $p$ is an approximate fibration.

Proof. If $p$ is an approximate fibration, the argument of Theorem 2.4 of [3] shows that $p$ is completely movable. Suppose that $p$ is completely movable. We shall show that $p$ is $k$-movable for all $k$ and apply 3.4. Let $b \in B$ be given and let $U_{0}$ be a neighborhood of $F_{b}$. Let $U_{1}$ be a movability choice for $U_{0}$ and $b$ and let $U_{2}$ be a movability choice for $U_{1}$ and $b$. We claim $U=U_{0}$ and $V=U_{1}$ satisfy the definition of $k$-movability for $U_{0}$ and $b$. To show this, let $c$ be such that $F_{c} \subset U_{2}$. Construct a sequence $\left\{U_{i}\right\}_{i=0}^{\infty}$ of neighborhoods of $F_{c}$ as follows. $U_{0}, U_{1}, U_{2}$ have been chosen. $U_{i}$, $i>2$ is chosen as a movability choice for $U_{i-1}$ and $c$ such that $\bigcap_{i=0}^{\infty} U_{i}=F_{c}$. Note that $U_{i}$ is also a movability choice for $U_{i-1}$ and $c, i=1,2$. Let $j_{i}: U_{i} \rightarrow U_{i-1}$ be the inclusion. First we show that for $i>1, \quad j_{i^{*}}: \pi_{k}\left(U_{i}\right) \rightarrow \pi_{k}\left(U_{i-1}\right)$ takes im $j_{i+1^{*}}$ isomorphically onto $\operatorname{im} j_{i^{*}}$ for each base point in $F_{\mathrm{c}}$. The proof that im $j_{i+1^{*}}$ maps onto im $j_{i^{*}}$ is an immediate consequence of the movability choices, so we only show that $j_{i^{*}}$ is $1-1$ on image $j_{i+1^{*}}$. To this end, let $\alpha: S^{k} \rightarrow U_{i+1}$ be a pointed map which represents the zero element in $\pi_{k}\left(U_{i-1}\right)$. Let $h_{t}$ be a homotopy of $U_{i-1}$ in $U_{i-2}$ which is fixed on $F_{c}$ and is such that $h_{0}=$ inclusion, $h_{1}\left(U_{i-1}\right) \subset U_{i+1}$. There is a neighborhood $W$ of $F_{c}$ such that $h_{t}(W) \subset U_{i+1}$ for all $t$. Using the movability choice of $U_{i+1}$, we can find a pointed map $\beta: S^{k} \rightarrow W$ such that $[\alpha]=[\beta]$ in $U_{i}$. There is a pointed homotopy $g_{t}: S^{k} \rightarrow U_{i-1}$ such that $g_{0}=\beta$, 
$g_{1}=$ constant map. Then $h_{1} g_{t}$ is a homotopy of $h_{1} \beta$ to a constant map in $U_{i+1}$ and $h_{t} \beta$ is a homotopy of $\beta$ to $h_{1} \beta$ in $U_{i+1}$. Thus $[\beta]=[\alpha]=0$ in $\pi_{k}\left(U_{i}\right)$. To complete the proof of the proposition, note that we have shown that the sequence $\cdots \rightarrow \pi_{k}\left(U_{i+1}\right) \rightarrow \pi_{k}\left(U_{i}\right) \rightarrow$ is constant, so that the inverse limit $\pi_{k}\left(F_{c}\right)$ projects isomorphically onto each image $j_{i^{*}}$ for each $i>1$.

Next we prove that $k$-movability up to the dimension of the fibers plus one is sufficient to get an approximate fibration.

THEOREM 3.7. If $p: E \rightarrow B$ is a $(k+1)$-movable map and $\operatorname{dim} F_{b} \leqq k$ for each fiber $F_{b}$, then $p$ is an approximate fibration.

Proof. We will show that $p$ is completely movable. Let $b \in B$. Since $p$ is a $(k+1)$-movable map, $F_{b}$ is a $(k+1)$-movable compactum by Lemma 3.2. Thus by [10, Theorem 4], $F_{b}$ is movable. Given a neighborhood $\widetilde{U}$ of $F_{b}$, we may assume that $\widetilde{U}=p^{-1}(U)$ for some neighborhood $U$ of $b$. By standard homotopy constructions, there is a neighborhood $U_{0}$ of $b$ in $U$ such that if $b^{\prime} \in U_{0}, U_{0}$ can be contracted to $b^{\prime}$ in $U$ keeping $b^{\prime}$ fixed. Let $\widetilde{U}_{0}=p^{-1}\left(U_{0}\right)$. By the movability of $F_{b}$ there is a neighborhood $\tilde{V}$ of $F_{b}$ in $\widetilde{U}_{0}$ so that $\tilde{V}$ deforms into each neighborhood of $F_{b}$ staying in $\widetilde{U}_{0}$. Again we may assume that $\widetilde{V}=p^{-1}(V)$, where $V$ is a compact connected neighborhood of $b$ in $U_{0}$. Let $b^{\prime} \in V$ be any point and let $W$ be any neighborhood of $b^{\prime}$ in $V$. It suffices to show that $\tilde{V}$ deforms into $\widetilde{W}=p^{-1}(W)$ in $\widetilde{U}$ keeping $F_{b^{\prime}}$ fixed. By our choice of $U_{0}$, there is a homotopy $K: U_{0} \times I \rightarrow U$ which fixes $b^{\prime}$ and such that $K_{0}=1_{U_{0}}$, $K_{1}\left(U_{0}\right)=b^{\prime}$. Let $W_{0}$ be a neighborhood of $b^{\prime}$ such that $K_{t}\left(W_{0}\right) \subset W$ for all $t$, and let $\widetilde{W}_{0}=p^{-1} W_{0}$. Let $\alpha: I \rightarrow V$ be a path from $b$ to $b^{\prime}$. Let $h: F_{b} \times I \rightarrow B$ be the homotopy given by $h(x, t)=\alpha(t)$, let $g: F_{b} \times\{0\} \rightarrow E$ be given by $g(x, 0)=x$, and let $G: F_{b} \times I \rightarrow \widetilde{V}$ be an approximate lifting of $h$ extending $g$ such that $G\left(F_{b} \times\{1\}\right) \subset \widetilde{W}_{0}$. By [1, IV, 8.1], there is a neighborhood $W^{\prime}$ of $F_{b}$ and an extension $G: W^{\prime} \times I \rightarrow \widetilde{V}$ such that $G\left(W^{\prime} \times 1\right) \subset \widetilde{W}_{0}$. Let $H: \widetilde{V} \times I \rightarrow \widetilde{U}_{0}$ be a homotopy such that $H_{0}=1_{\tilde{V}}$ and $H_{1}(\widetilde{V}) \subset W^{\prime}$. Then $\phi: \widetilde{V} \times I \rightarrow \widetilde{U}_{0}$ given by

$$
\dot{\phi}(x, t)= \begin{cases}H(x, 2 t), & 0 \leqq t \leqq \frac{1}{2} ; \text { and } \\ G\left(H_{1}(x), 2 t-1\right), & \frac{1}{2} \leqq t \leqq 1\end{cases}
$$

is a homotopy of $\tilde{V}$ which deforms $\tilde{V}$ into $\tilde{W}$ in $\tilde{U}_{0}$. It remains to alter $\phi$ so as to hold $F_{b^{\prime}}$ fixed. Define $f: F_{b^{\prime}} \times I \times I \rightarrow U$ by 


$$
f(x, t, s)=K(p \phi(x, t), s)
$$

and let $\widetilde{f}: F_{b^{\prime}} \times I \rightarrow E$ be defined by $\widetilde{f}=\phi \mid\left(F_{b^{\prime}} \times I\right)$. By our choice of $\widetilde{W}_{0}$, we can find an approximate lifting $\widetilde{K}: F_{b^{\prime}} \times I \times I \rightarrow \widetilde{U}$ of $f$ which extends $\widetilde{f}$ and such that $\widetilde{K}\left(F_{b}, \times I \times\{1\}\right) \subset \widetilde{W}$ and $\widetilde{K}\left(F_{b^{\prime}} \times\{1\} \times I\right) \subset \hat{W}$. By stationary lifting we may make $\widetilde{K}(x, 0, s)=x$ for all $s$.

By reparameterizing $I \times I$, we can use $\widetilde{K}$ to get a map $K^{\prime \prime}: F_{b^{\prime}} \times$ $I \times I \rightarrow \widetilde{U}$ such that $K^{\prime \prime}(x, t, 0)=\phi(x, t), K^{\prime \prime}(x, t, s)=x$ if $t=0$ or $s=1$, and $K^{\prime \prime}(x, 1, s) \in \bar{W}$ for all $t, x \in F_{b^{\prime}}$.

Consider the map $\phi^{\prime}: \tilde{V} \times\{1\} \times\{0\} \cup F_{b^{\prime}} \times\{1\} \times I \rightarrow \widetilde{W}$ given by $\phi^{\prime}(x, 1, s)=\left\{\begin{array}{ll}\phi(x, 1), & s=0 \\ K^{\prime \prime}(x, 1, s), & x \in F_{b^{\prime}} .\end{array}\right.$ By [1, IV, 8.1] $\phi^{\prime}$ extends to a map $\phi^{\prime}: \tilde{V} \times\{1\} \times I \rightarrow \widetilde{W}$. Now define

$$
\phi^{\prime \prime}:(\tilde{V} \times I \times\{0\}) \cup(\widetilde{V} \times\{0,1\} \times I) \cup\left(F_{b}^{\prime}, \times I \times I\right) \longrightarrow \widetilde{U}
$$

by

$$
\phi^{\prime \prime}(x, t, s)= \begin{cases}\phi(x, t), & s=0 ; \\ \phi^{\prime}(x, 1, s) & t=1 ; \\ x, & t=0 ; \quad \text { and } \\ K^{\prime \prime}(x, t, s), & x \in F_{b^{\prime}} .\end{cases}
$$

By [1] again, $\phi^{\prime \prime}$ extends to $\phi^{\prime \prime}: \widetilde{V} \times I \times I \rightarrow \widetilde{U}$. Then $\psi: \widetilde{V} \times I \rightarrow \widetilde{U}$, given by $\psi(x, t)=\phi^{\prime \prime}(x, t, 1)$ is a homotopy which deforms $\widetilde{V}$ into $\widetilde{W}$ in $\widetilde{U}$ keeping $F_{b}$, fixed.

Proposition 3.8. Let $p: E \rightarrow B$ be a proper map. If $p$ is a $k$ movable map and $a(k+1)-U V \operatorname{map}, k \geqq-1$, then $p$ is $a(k+1)$ movable map.

Proof. Given a neighborhood $U_{0}$ of some fiber $F_{b}$ choose neighborhoods $U$ and $V$ of $F_{b}$ in $U_{0}$ such that each singular $(k+1)$-sphere in $V$ is null-homotopic in $U$. Then $\pi_{k+1} F_{c}$ is realized as the image of $\pi_{k+1}(V)$ in $\pi_{k+1}(U)$ for any fiber $F_{c} \subset V$ since both are zero.

Finally, we summarize the results of this and the previous section:

TheOReM 3.9. For $p: E \rightarrow B$ a proper map between locally compact, separable ANR's, the following are equivalent:

(1) $p$ is completely movable,

(2) $p$ is an approximate fibration, 
(3) $p$ is k-movable for all $k$, and

(4) $p$ has the AHLP for $I^{q}, 0 \leqq q<\infty$.

IV. An application. In this section, we combine the results of $\S \S 2$ and 3 with the geometric results of L. Husch in [8] to give some conditions under which a map between manifolds can be approximated by locally trivial fibrations.

THEOREM 4.1. Suppose $E$ is a closed connected 3-manifold such that each innessential tame 2-sphere in $E$ bounds a 3-cell and let $B$ be a connected 2-manifold. If $f: E \rightarrow B$ is a surjective map such that $f$ is 1-movable and each fiber of $f$ has fundamental dimension less than or equal to 1 , then $f$ is the uniform limit of locally trivial fiber maps.

Proof. Each fiber has property $k-U V$ for $k \geqq 2$ since each fiber has a shape representative $X$ in the Menger universal curve and we can use $[15, \mathrm{~L} .2]$ to write $X$ as the intersection of cubes-with handles. Hence, $f$ is an approximate fibration by $3.9,3.3$, and 3.4. The theorem thus follows from [8, Theorem A].

THEOREM 4.2. Let $f: E \rightarrow S^{1}$ be an $n+1$-movable map where $E$ is a closed, connected $n$-manifold, $n=3$ or $n \geqq 6$. If $n=3$ suppose that each tame innessential 2-sphere in $E$ bounds a 3-cell and that for some $b \in S^{1}, \pi_{1}\left(F_{b}\right) \neq Z_{2}$; if $n \geqq 6$, suppose that for some $b \in S^{1}$, the Whitehead and projective class groups of $\pi_{1}\left(F_{b}\right)$ are trivial. Then $f$ can be uniformly approximated by locally trivial fibrations.

Proof. By 3.7 and $3.4 f$ is an approximate fibration, so the theorem follows by [8, Theorems $\mathrm{A}$ and $\mathrm{B}]$.

Finally, we give a characterization of the $n$-sphere, $n \geqq 5$, similar to McAuley's version of the Reeb-Milnor theorem [14].

THEOREM 4.3. Suppose $M^{n}$ is a closed (connected) manifold, $n \geqq 5$, and $f: M^{n} \rightarrow[0,1]$ is a surjective map such that $f \mid f^{-1}(0,1)$ $i$ completely movable and $f^{-1}(i)$ is $U V^{\infty}$ for $i=0,1$. Then $M^{n}$ is homeomorphic to $S^{n}$.

Proof. Let $A=f^{-1}(0), B=f^{-1}(1)$. We first show that $M-A$ and $M-B$ are contractible. Since $A$ is $U V^{\infty}$, there exists an $\varepsilon>0$ so that the inclusion of $f^{-1}([0, \varepsilon))$ into $M-B$ is null-homotopic. By Proposition 3.6, $f \mid M-(A \cup B)$ is an approximate fibration. Let $h_{t}$ be a homotopy of $(0,1)$ such that $h_{0}=$ identity, $h_{1}(0,1) \subset(0, \varepsilon)$, 
and $h_{t}$ is stationary on some interval $(0, \delta) \subset(0, \varepsilon)$. Let

$$
g:(M-(A \cup B)) \times\{0\} \longrightarrow M-(A \cup B)
$$

be given by $g(x, 0)=x, G:(M-(A \cup B)) \times I \rightarrow(0,1)$ by $G(x, t)=$ $h_{t} f(x)$. By choosing a suitable stationary approximate lift of $G$, we get a homotopy $H_{t}$ of $M-(A \cup B)$ which deforms $M-(A \cup B)$ into $f^{-1}(0, \varepsilon)$ and is stationary on some neighborhood of $A$. Thus $H_{t}$ extends to a deformation of $M-B$ into $f^{-1}([0, \varepsilon))$ so $M-B$ is contractible. A similar argument shows that $M-A$ is contractible. Since $M-(A \cup B)$ is connected by duality, $M$ is 1-connected by the Van Kampen theorem. To complete the proof, we need only show that $M$ has the homology of $S^{n}$. By duality,

$$
H_{k}(M-A, M-(A \cup B)) \cong \check{H}^{n-k}(A \cup B, A) \cong \begin{cases}Z, & k=n \\ 0, & k \neq n\end{cases}
$$

Then $M-(A \cup B)$ has the homology of $S^{n-1}$ by the homology sequence of the pair $(M-A, M-(A \cup B))$, and $M$ has the homology of $S^{n}$ by the Mayer-Vietoris sequence of $(M, M-A, M-B)$.

The authors thank J. Maxwell for a helpful discussion concerning Theorem 4.3.

\section{REFERENCES}

1. K. Borsuk, Theory of Retracts, Polish Scientific Publishers, Warsaw, 1967.

2. - On the n-movability, Bull. Acad. Polon. Sci. Sér. Sci. Math. Astrom. Phys., 20 (1972), 859-864.

3. D. Coram and P. Duvall, Approximate fibrations, Rocky Mountain J. Math., (to appear).

4. J. Dugundji, Topology, Allyn and Bacon, Boston, 1966.

5. E. Dyer and M. E. Hamstrom, Completely regular mappings, Fund. Math., 45 (1957), 103-118.

6. S. Eilenberg and N. Steenrod, Foundations of Algebraic Topology, Princeton University Press, Princeton, 1952.

7. S. T. Hu, Theory of Retracts, Wayne State University Press, Detroit, 1965.

8. L. Husch, Approximating approximate fibrations by fibrations, (to appear).

9. G. Kozlowski, Factorization of certain maps up to homotopy, Proc. Amer. Math. Soc., 21 (1969), 88-92.

10. G. Kozlowski and J. Segal, n-movable compacta and ANR-systems, Fund. Math., 85 (1974), 235-243.

11. R. C. Lacher, Cellularity criteria for maps, Mich. Math. J., 17 (1970), 385-396.

12. Cell-like mappings II, Pacific J. Math., 35 (1970), 649-660.

13. L. McAuley, Proceedings of the Conference on Monotone Mappings and Open Mappings 1970, State University of New York at Binghamton.

14. —, A topological Reeb-Milnor-Rosen theorem and characterizations of manifolds, Bull. Amer. Math. Soc., 78 (1972), 82-84.

15. D. R. McMillan, Jr., A criterion for cellularity in a manifold II, Trans. Amer. Math. Soc., 126 (1976), 217-224. 
16. M. Moszyńska, Various approaches to the fundamental groups, Fund. Math., 78 (1973), 107-118.

17. Stephen B. Seidman, Completely regular mappings with locally compact fiber, Trans. Amer. Math. Soc., 147 (1970), 461-471.

18. - Completely regular mappings with compact ANR fiber, Fund. Math., 70 (1971), 139-146.

19. E. Spanier, Algebraic Topology, McGraw-Hill, New York, 1966.

Received May 3, 1976. Research of the second author supported by NSF Grant MPS-75-07084.

OKLahoma STAte University

STILLWATER, OK 74074 


\title{
PACIFIC JOURNAL OF MATHEMATICS
}

\author{
EDITORS
}

RICHARD ARENS (Managing Editor)

University of California

Los Angeles, California 90024

C. W. CurTis

University of Oregon

Eugene, OR 97403

C. C. MOORE

University of California

Berkeley, CA 94720
J. DUgundJI

Department of Mathematics

University of Southern Californı

Los Angeles, California 90007

R. Finn and J. Milgram

Stanford University

Stanford, California 94305

\section{ASSOCIATE EDITORS}

E. F. BECKENBACH

\section{SUPPORTING INSTITUTIONS}

\author{
UNIVERSITY OF BRITISH COLUMBIA \\ CALIFORNIA INSTITUTE OF TECHNOLOGY \\ UNIVERSITY OF CALIFORNIA \\ MONTANA STATE UNIVERSITY \\ UNIVERSITY OF NEVADA, RENO \\ NEW MEXICO STATE UNIVERSITY \\ OREGON STATE UNIVERSITY \\ UNIVERSITY OF OREGON \\ OSAKA UNIVERSITY
}

UNIVERSITY OF SOUTHERN CALIFORNIA

STANFORD UNIVERSITY

UNIVERSITY OF TOKYO

UNIVERSITY OF UTAH

WASHINGTON STATE UNIVERSITY

UNIVERSITY OF WASHINGTON
AMERICAN MATHEMATICAL SOCIETY
NAVAL WEAPONS CENTER 


\section{Pacific Journal of Mathematics \\ Vol. 72 , No. 1 \\ January, 1977}

Kazuo Anzai and Shiro Ishikawa, On common fixed points for several

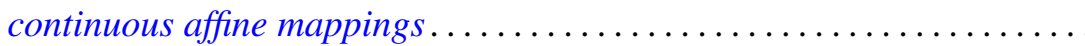

Bruce Alan Barnes, When is a representation of a Banach $*$-algebra

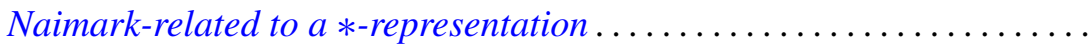

Richard Dowell Byrd, Justin Thomas Lloyd, Franklin D. Pedersen and

James Wilson Stepp, Automorphisms of the semigroup of finite

complexes of a periodic locally cyclic group ...................

Donald S. Coram and Paul Frazier Duvall, Jr., Approximate fibrations and a

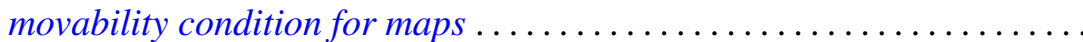

Kenneth R. Davidson and Che-Kao Fong, An operator algebra which is not

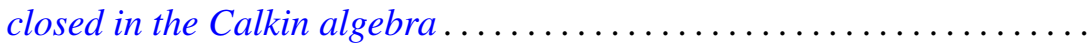

Garret J. Etgen and James Pawlowski, A comparison theorem and oscillation criteria for second order differential systems .............

Philip Palmer Green, $C^{*}$-algebras of transformation groups with smooth

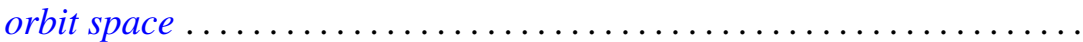

Charles Allen Jones and Charles Dwight Lahr, Weak and norm approximate

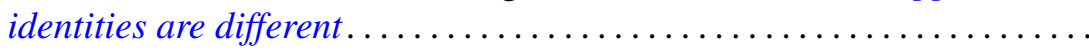

G. K. Kalisch, On integral representations of piecewise holomorphic

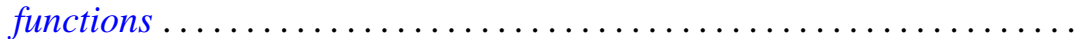

Y. Kodama, On product of shape and a question of Sher

Heinz K. Langer and B. Textorius, On generalized resolvents and

$Q$-functions of symmetric linear relations (subspaces) in Hilbert

space ...................................

Albert Edward Livingston, On the integral means of univalent, meromorphic functions

Wallace Smith Martindale, III and Susan Montgomery, Fixed elements of

Jordan automorphisms of associative rings ..........

R. Kent Nagle, Monotonicity and alternative methods for nonlinear boundary value problems ........................

Richard John O'Malley, Approximately differentiable functions: the $r$ topology.

Mangesh Bhalchandra Rege and Kalathoor Varadarajan, Chain conditions

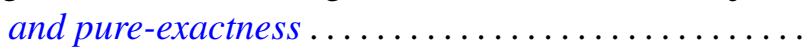

Christine Ann Shannon, The second dual of $C(X)$. .

Sin-ei Takahasi, A characterization for compact central double centralizers of $C^{*}$-algebras .

Theresa Phillips Vaughan, A note on the Jacobi-Perron algorithm. . .

Arthur Anthony Yanushka, A characterization of $\operatorname{PSp}(2 m, q)$ and

$\mathrm{P} \Omega(2 m+1, q)$ as rank 3 permutation groups ......... 